\title{
Symmetries of mesons after unbreaking of chiral symmetry and their string interpretation
}

\author{
M. Denissenya.* L.Ya. Glozman $\left.\right|^{\dagger}$ and C.B. Lang ${ }^{\dagger}$ \\ Institute of Physics, University of Graz, A-8010 Graz, Austria
}

\begin{abstract}
Using the chirally invariant overlap Dirac operator we remove its lowest-lying quasizero modes from the valence quark propagators and study evolution of isovector mesons with $J=1$. At the truncation level about $50 \mathrm{MeV} S U(2)_{L} \times S U(2)_{R}$ and $U(1)_{A}$ symmetries get restored. However, we observe a degeneracy not only within the chiral and $U(1)_{A}$ multiplets, but also a degeneracy of all possible chiral multiplets, i.e., the observed quantum levels have a symmetry larger than $U(2)_{L} \times U(2)_{R}$ and their energy does not depend on the spin orientation of quarks and their parities. We offer a possible interpretation of these energy levels as the quantum levels of the dynamical QCD string. The structure of the radial $J=1$ spectrum is compatible with $E=\left(n_{r}+1\right) \hbar \omega$ with $\hbar \omega=900 \pm 70 \mathrm{MeV}$.

PACS numbers: 11.30.Rd, 12.38.Aw, 11.25.-w
\end{abstract}

1. Introduction. A consistent and systematic picture of hadrons made of light quarks is missing. There is a general understanding that both confinement and spontaneous breaking of chiral symmetry $(\mathrm{SB} \chi \mathrm{S})$ are important for hadronic mass generation. A large degeneracy is seen in the highly excited mesons [1, 2], which is however absent in the observed spectrum of mesons with masses below $1.7-1.8 \mathrm{GeV}$. The physics of the low-lying hadrons should be affected by the $\mathrm{SB} \chi \mathrm{S}$ that might obscure the primary confinement picture. We want to disentangle the primary physics that is responsible for a genesis of hadrons from the $\mathrm{SB} \chi \mathrm{S}$.

In order to address this issue we artificially remove the chiral symmetry breaking dynamics from the valence quark propagators keeping at the same time the gluonic gauge configurations intact [3 5]. It has been known for a long time that the quark condensate of the vacuum is directly related to the density of the close-to-zero modes of the Dirac operator [6]. The quark propagator can be written in terms of projectors onto the eigenmodes of the Dirac operator. We subtract from the full propagator its lowest-lying modes, that represent only a tiny part of the full amount of modes,

$$
S_{R D(k)}=S_{\text {Full }}-\sum_{i=1}^{k} \frac{1}{\lambda_{i}}\left|\lambda_{i}\right\rangle\left\langle\lambda_{i}\right|,
$$

where $\lambda_{i}$ and $\left|\lambda_{i}\right\rangle$ are the eigenvalues and the corresponding eigenvectors of the Dirac operator. This way we artificially restore ("unbreak") the chiral symmetry.

In ref. 4] we have noticed that after unbreaking the chiral symmetry a degeneracy of hadrons develops that is higher than $S U(2)_{L} \times S U(2)_{R}$. Such a degeneracy should reflect the underlying symmetry and dynamics. Still the quality of our data that support a degeneracy was not too high.

A reason for not a high quality was that in Refs. 3 5] a not-chirally-symmetric lattice Dirac operator of the Wilson type was used. An eigenvalue decomposition in terms of the Hermitian Dirac operator $\gamma_{5} D$ was adopted. It causes ambiguity as there is no direct bijective relation between the low-lying modes of $\gamma_{5} D$ and of the Dirac operator $D$; exact zero modes could not be exactly identified. In the present work we use the manifestly chirally invariant overlap Dirac operator [7] where such problems are absent.

From the truncated quark propagators we construct hadron propagators. When we observe an exponential decay signal of the corresponding correlation function we interpret it as a physical state and extract its mass. Our task is to see what will happen with hadrons and their masses when we subtract the lowest-lying modes of the Dirac operator.

2. Lattice technology. We adopt 100 gauge field configurations generated with $n_{f}=2$ dynamical overlap fermions on a $16^{3} \times 32$ lattice with the spacing $a \sim 0.12$ $\mathrm{fm}$ [8, 9. The pion mass in this ensemble is $M_{\pi}=289(2)$ $\mathrm{MeV}$ [10]. For each hadron we use a set of up to eight interpolators with different Dirac structures (see Table I) and different exponential smearings of sources and sinks with several smearing widths. We determine correlators for all polarizations. The quark propagators were generously provided by the JLQCD collaboration and were computed by combining of the exact 100 low modes with stochastic estimates for the higher modes 9 .

For the analysis of the cross-correlation matrices we employ the variational method and solve the generalized eigenvalue problem 11. In the figures we show effective energy values (nearest neighbour slopes) to demonstrate plateau behaviour. The horizontal bars on the effective energy plots indicate the fit range. The one-exponential fits are to the eigenvalues of the correlation matrices in the range shown in the effective mass plots. The error bars are determined by the single-elimination jackknife method. Table [I] gives the resulting numbers for two stages of truncation, $k=10,20$.

3. Observations. We have studied the ground state and the excited states of all possible $\bar{q} q$ isovector $J=1$ 
TABLE I: Interpolators for the $\rho\left(1^{--}\right), a_{1}\left(1^{++}\right)$and $b_{1}\left(1^{+-}\right)$ mesons $(i, j=1,2,3)$ with 7 different smearing widths at sink/source.

\begin{tabular}{ccc}
\hline \hline channel & interpolator types & no. of interpolators \\
\hline$\rho$ & $\bar{q} \gamma_{i} \vec{\tau} q, \bar{q} \gamma_{i} \gamma_{t} \vec{\tau} q$ & 8 \\
$a_{1}$ & $\bar{q} \gamma_{i} \gamma_{5} \vec{\tau} q$ & 5 \\
$b_{1}$ & $\bar{q} \gamma_{i} \gamma_{j} \vec{\tau} q$ & 6 \\
\hline \hline
\end{tabular}

TABLE II: Masses of the $\rho, \rho^{\prime}, a_{1}, b_{1}$ mesons extracted at the specific truncation levels $k$.

\begin{tabular}{ccccc}
\hline \hline & \multicolumn{2}{c}{$k=10$} & \multicolumn{2}{c}{$k=20$} \\
\hline$a m$ & $n_{r}=0$ & $n_{r}=1$ & $n_{r}=0$ & $n_{r}=1$ \\
\hline$\rho$ & $0.573(10)$ & $1.096(57)$ & $0.625(7)$ & $1.107(58)$ \\
$\rho^{\prime}$ & $0.589(13)$ & $1.108(48)$ & $0.614(8)$ & $1.139(48)$ \\
$a_{1}$ & $0.576(10)$ & $1.051(62)$ & $0.615(8)$ & $1.062(61)$ \\
$b_{1}$ & $0.568(14)$ & $1.077(51)$ & $0.607(8)$ & $1.106(51)$ \\
\hline \hline
\end{tabular}

mesons, i.e., $\rho\left(1^{--}\right), a_{1}\left(1^{++}\right), b_{1}\left(1^{+-}\right)$. Typical results for the eigenvalues of the correlation matrices and the effective mass plots are shown in Fig. 1. Upon removal of the lowest eigenmodes of the Dirac operator from the valence quark propagators we observe an improving signal for clean exponential decay. The evolution of masses of the ground and excited states are shown in Fig. 2 . Around a truncation energy of 40-65 MeV (approximately 10 eigenmodes in our ensemble) an onset of a degeneracy of four states, $\rho, \rho^{\prime}, a_{1}, b_{1}$, as well as a degeneracy of their excited states is seen [19]. The degeneracy indicates a yet unknown symmetry.

Before discussing the symmetry issue we want to exclude a possibility that the observed energy levels are levels of a free quark-antiquark pair in a box. For a system of a free quark and antiquark the lowest level would represent the energy of the quark and antiquark with the $p=0$ momentum, which is compatible with the $S-$ and incompatible with the $P$-wave of relative motion. The next level would correspond to $p=2 \pi / L$ ( $L$ is the spatial box size) for each quark and is compatible with both $S$ - and $P$-waves. Consequently, a system of a free quark and antiquark in a box cannot produce degenerate ground states of opposite parities. We do observe levels of a nontrivially bound (confined) quark-antiquark system.

4. Symmetries. Which symmetries does the observed degeneracy represent? All possible $S U(2)_{L} \times S U(2)_{R}$ multiplets of the $J=1$ mesons are listed in Table III [1, 2].

When chiral symmetry is restored but the states still exist we expect that all mesons will fall into multiplets of the chiral group and within each independent chiral multiplet the mesons will be degenerate. In the chirally sym-
TABLE III: The complete set of $q \bar{q} J=1$ states classified according to the chiral basis. The symbol $\leftrightarrow$ indicates the states belonging to the same representation $R$ of $S U(2)_{L} \times$ $S U(2)_{R}$ that must be degenerate in the $S U(2)_{L} \times S U(2)_{R}$ symmetric world.

\begin{tabular}{cc}
\hline \hline$R$ & mesons \\
\hline$(0,0)$ & $\omega\left(I=0,1^{--}\right) \leftrightarrow f_{1}\left(I=0,1^{++}\right)$ \\
$(1 / 2,1 / 2)_{a}$ & $\omega\left(I=0,1^{--}\right) \leftrightarrow b_{1}\left(I=1,1^{+-}\right)$ \\
$(1 / 2,1 / 2)_{b}$ & $h_{1}\left(I=0,1^{+-}\right) \leftrightarrow \rho\left(I=1,1^{--}\right)$ \\
$(0,1) \oplus(1,0)$ & $a_{1}\left(I=1,1^{++}\right) \leftrightarrow \rho\left(I=1,1^{--}\right)$ \\
\hline \hline
\end{tabular}

metric world there must be two independent $\rho$-mesons. One of them is a member of the $(0,1) \oplus(1,0)$ multiplet and can be created only by an operator with the same chiral structure, e.g., by the vector current, and that should be degenerate with its chiral partner $a_{1}$. Another $\rho$-meson forms together with its chiral partner $h_{1}$ the $(1 / 2,1 / 2)_{b}$ representation and is coupled only to an operator of the type $\bar{q} \sigma^{0 i} \vec{\tau} q$.

A degeneracy of the $(0,1) \oplus(1,0) \rho$-meson with the $a_{1}$ meson is a clear signal of the chiral $S U(2)_{L} \times S U(2)_{R}$ restoration. We have not yet studied the isoscalar mesons, since they contain disconnected graphs and their observation represents a challenge for the simulation. An observation of the degeneracy within one of the multiplets is sufficient to confirm chiral restoration, however. Consequently, a similar degeneracy should be seen in other chiral pairs.

The $U(1)_{A}$ symmetry connects the $(1 / 2,1 / 2)_{b} \rho$-meson with the $b_{1}$ meson [1, 2]. At a truncation energy around $50 \mathrm{MeV}$ the onset of this degeneracy is also seen. We conclude that simultaneously both $S U(2)_{L} \times S U(2)_{R}$ and $U(1)_{A}$ symmetries get restored [20]. Both chiral and $U(1)_{A}$ breaking are produced by the same low-lying modes of the Dirac operator which is consistent with the instanton-induced mechanism $12 \sqrt{14}$.

The restored $S U(2)_{L} \times S U(2)_{R} \times U(1)_{A}$ symmetry requires a degeneracy of four mesons that belong to $(1 / 2,1 / 2)_{a}$ and $(1 / 2,1 / 2)_{b}$ chiral multiplets [1, 2], see Table III. This symmetry does not require, however, a degeneracy of these four states with other mesons, in particular with $a_{1}$ and its chiral partner $\rho$. We clearly see the latter degeneracy. This implies that there is some higher symmetry, that includes $U(2)_{L} \times U(2)_{R}$ as a subgroup. While a degeneracy of all isovector mesons is sufficient to claim this, this higher symmetry requires also a degeneracy of all mesons in Table III, which is a task for future studies.

One could ask whether this situation is similar or not to the high temperature deconfining regime, where chiral and $U(1)_{A}$ symmetries are also restored. In the latter case correlators of the chiral partners become also identical. This identity does not imply, however, that there is a complete spectrum of the quark-antiquark bound ground 

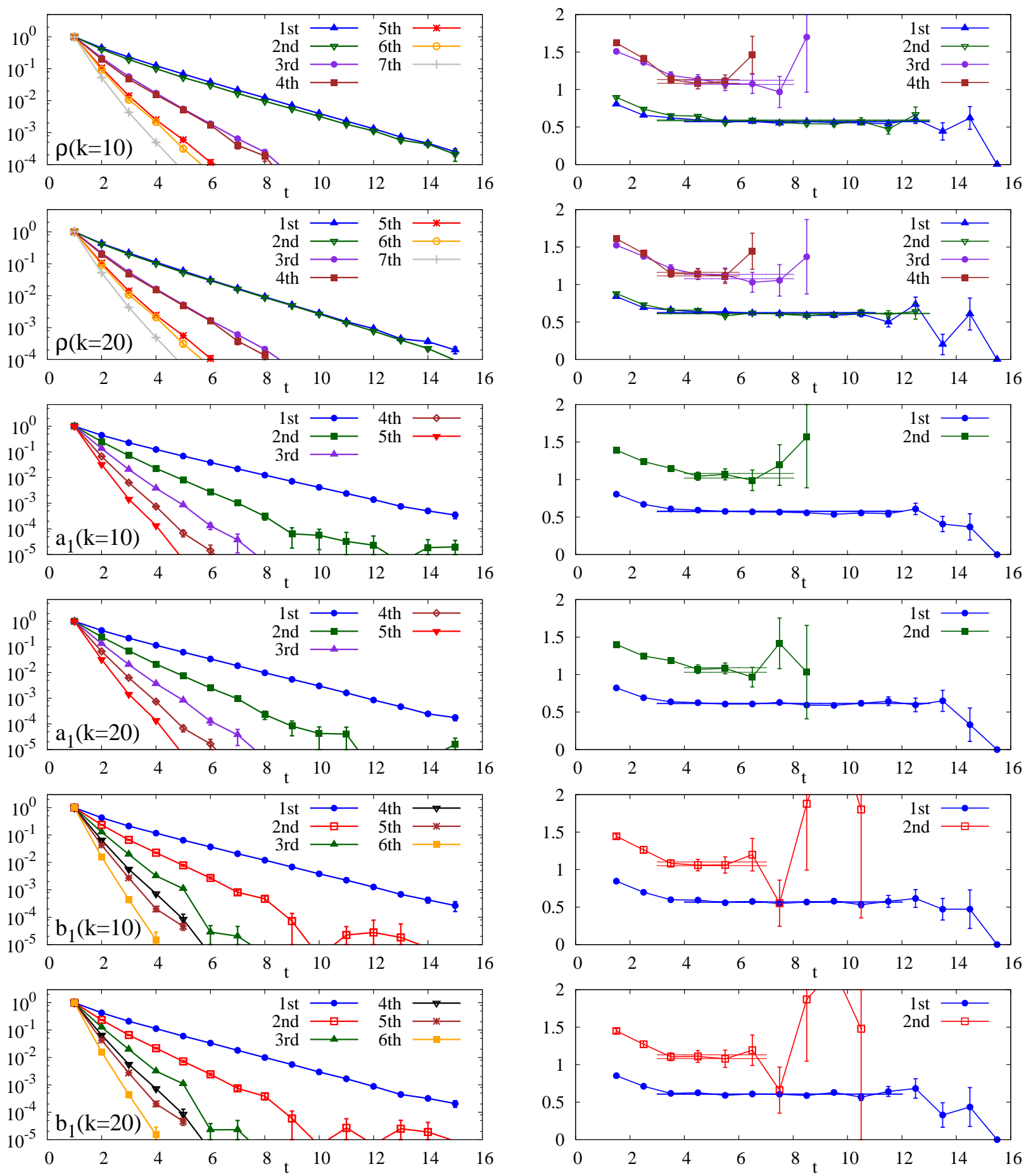

FIG. 1: Upper panel: $\rho$-meson $\left(J^{P C}=1^{--}\right)$, middle part: $a_{1}$-meson $\left(J^{P C}=1^{++}\right)$, lower panel: $b_{1}$-meson $\left(J^{P C}=1^{+-}\right)$. We show eigenvalues of the correlation matrix (the left-hand plots) and effective masses (the right-hand plots) for $k=10$ and $k=20$ for each meson, respectively. Please note the degeneracy of the levels of the $\rho$ meson, different from the untruncated case.

and excited states. The high temperature affects the gluodynamics. In our case, in contrast, the gluodynamics is kept intact. The valence quarks have no effect on the Polyakov and Wilson loops as well as on the potential between the static infinitely heavy quarks.
5. Our interpretation. The $Q C D$ dynamical string. It was suspected for a long time and illustrated within the abelian models that the colored quarks are connected by the color-electric flux tube which, if long enough, can be approximated by a string. This picture has obtained 


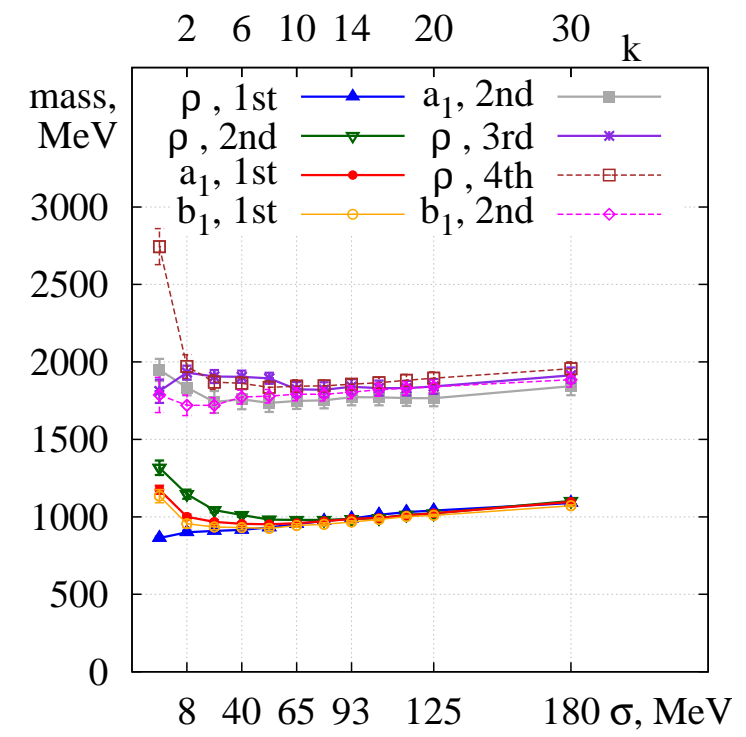

FIG. 2: Evolution of hadron masses under the low-mode truncation. Both the number $k$ of the removed lowest eigenmodes as well as the corresponding energy gap $\sigma$ are given

some support from the lattice simulations with the static quark sources, where a flux tube as well as the linear potential have been observed (for review and references see [15]). That string is nondynamical (in the sense that its ends are fixed), however, and can only illustrate to some extent physics of hadrons made of heavy quarks. In the light quark sector the fast motion of quarks at the ends of a possible string, the respective chiral symmetry as well as its dynamical breaking should be of great importance. In this case it is even apriori unclear whether the stringy picture has something to do with reality. There is no consistent theory of the QCD string with quarks at the ends.

The energy of the string with unbroken chiral symmetry is stored in the gluonic color-electric flux tube, that is created by the color charges of quarks at the ends of the string. This energy should not depend on the orientation of the quark spins, because the quark spins can interact only with the color-magnetic fields 16. Consequently, one expects that the energy of the system at a given $J$ should be the same for all possible orientations of the quark spins and their parities.

All eight mesons in Table III can be combined into a reducible chiral representation [17]:

$$
\begin{aligned}
& {[(0,1 / 2)+(1 / 2,0)] \times[(0,1 / 2)+(1 / 2,0)]=} \\
& (0,0)+(1 / 2,1 / 2)_{a}+(1 / 2,1 / 2)_{b}+[(0,1)+(1,0)] .
\end{aligned}
$$

They exhaust all possible chiralities of quarks and antiquarks, i.e., their spin orientations, as well as possible spatial and charge parities for non-exotic mesons. The observed degeneracy of all these mesons suggests that we see the energy levels of the dynamical QCD string that connects the ultra-relativistic quark and antiquark with the total spin $J=1$.

In Fig. 2 we show the two lowest energy levels. We actually get also a signal of the third level, as can be seen from the eigenvalues of the correlation matrices on Fig. 1. The quality the third level is not sufficient to show this level in Fig. 2.

The observed radial levels at the truncation energy, at which we see the onset of the symmetry, are approximately equidistant and are compatible with the simple relation

$$
E_{n_{r}}=\left(n_{r}+1\right) \hbar \omega, \quad n_{r}=0,1, \ldots
$$

The extracted value of the fundamental string excitation quantum at the truncation energy $65 \mathrm{MeV}(k=10)$ amounts to $\hbar \omega=(900 \pm 70) \mathrm{MeV}$ [21]. In order to include into this quantization law the rotational levels one should study mesons with $J \neq 1$, which is planned.

6. Is this string of the Nambu-Goto type? A principal result for the classical Nambu-Goto open string is that the energy of the string is described in terms of its orbital angular momentum $L$ (which is a conserved quantum number): $M^{2} \sim L$. For the string with chiral quarks at the ends the orbital angular momentum $L$ of the relative motion of the quark and antiquark is not a conserved quantum number, though [18]. For instance, two independent $\rho$-mesons at the same energy level are represented by the mutually orthogonal fixed superpositions of the $S$ - and $D$-waves.

$$
\begin{aligned}
\left|(0,1)+(1,0) ; 11^{--}\right\rangle & =\sqrt{\frac{2}{3}}\left|1 ;{ }^{3} S_{1}\right\rangle+\sqrt{\frac{1}{3}}\left|1 ;{ }^{3} D_{1}\right\rangle, \\
\left|(1 / 2,1 / 2)_{b} ; 11^{--}\right\rangle & =\sqrt{\frac{1}{3}}\left|1 ;{ }^{3} S_{1}\right\rangle-\sqrt{\frac{2}{3}}\left|1 ;{ }^{3} D_{1}\right\rangle .
\end{aligned}
$$

Hence a description of a dynamical string with chiral quarks at the ends is impossible in terms of a fixed $L$. The total angular momentum $J$ is a conserved quantum number, of course, as required by Poincare-invariance.

We are deeply grateful to S. Aoki, S. Hashimoto and T. Kaneko for their suggestion to use the JLQCD overlap gauge configurations and quark propagators, for their help and hospitality during our visit to KEK. Support from the Austrian Science Fund (FWF) through the grants DK W1203-N16 and P26627-N16 is acknowledged.

* Electronic address: mikhail.denissenya@uni-graz.at

† Electronic address: leonid.glozman@uni-graz.at

¥ Electronic address: christian.lang@uni-graz.at

[1] L. Y. Glozman, Phys. Lett. B 587, 69 (2004).

[2] L. Y. Glozman, Phys. Rept. 444, 1 (2007).

[3] C. B. Lang and M. Schröck, Phys. Rev. D 84, 087704 (2011).

[4] L. Y. Glozman, C. B. Lang and M. Schröck, Phys. Rev. D 86, 014507 (2012). 
[5] M. Denissenya and L. Y. Glozman, arXiv:1401.6034 [heplat].

[6] T. Banks and A. Casher, Nucl. Phys. B 169, 103 (1980).

[7] H. Neuberger, Phys. Lett. B 417, 141 (1998).

[8] S. Aoki et al. [JLQCD Collaboration], Phys. Rev. D 78, 014508 (2008).

[9] S. Aoki et al. PTEP 2012, 01A106 (2012).

[10] J. Noaki et al. [JLQCD and TWQCD Collaborations], Phys. Rev. Lett. 101, 202004 (2008)

[11] M. Lüscher and U. Wolff, Nucl. Phys. B 339, 222 (1990); C. Michael, Nucl. Phys. B 259, 58 (1985); B. Blossier, M. DellaMorte, G. von Hippel, T. Mendes and R. Sommer, JHEP 04, 094 (2009).

[12] G. 't Hooft, Phys. Rept. 142, 357 (1986).

[13] E. V. Shuryak, Nucl. Phys. B 203, 93 (1982).

[14] D. Diakonov and V. Y. Petrov, Nucl. Phys. B 272, 457 (1986).

[15] G. S. Bali, Phys. Rept. 343, 1 (2001).

[16] L. Y. Glozman, Phys. Lett. B 541, 115 (2002).

[17] T. D. Cohen and X. -D. Ji, Phys. Rev. D 55, 6870 (1997).

[18] L. Y. Glozman and A. V. Nefediev, Phys. Rev. D 76, 096004 (2007); Phys. Rev. D 80, 057901 (2009).

[19] Note, that in order to observe two degenerate indepen- dent orthogonal $\rho$-mesons it is necessary to have in the correlation matrix operators with two different chiral structures, see the subsequent discussion. With the vector current type operator $\bar{q} \gamma_{\mu} \vec{\tau} q$ alone one of these states will not be seen, as that second $\rho$-meson does not couple to the vector current [1, 2].

[20] In our previous study with the Wilson-type Dirac operator 4 we claimed a non-restoration of the $U(1)_{A}$ symmetry. We attributed the reason to the unclear contribution of zero modes in that not exactly chirally symmetric Dirac operator. Given our present results obtained with the overlap Dirac operator, we have revisited our old analysis of the $b_{1}$ meson and traced an inadequate choice of the fit interval. Upon reanalysis the result is consistent to the present one.

[21] Approximating the observed levels with the quadratic relation $E_{n_{r}}^{2} \sim\left(n_{r}+1\right)$ leads to significantly larger deviations. The excited levels might be strongly affected by the finite lattice volume. Consequently, simulations with larger volumes are required in order to exclude or establish a linear or quadratic dependence. 